\title{
Spectral bounds on closed hyperbolic 3-manifolds
}

\author{
Nina White
}

\section{Abstract}

Fixing constants $\varepsilon$ and $c$, we consider the class of all closed $\varepsilon$-thick hyperbolic 3-manifolds $M$ such that $\pi_{1}(M)$ can be generated by $c$ elements. For all $k$, we prove that $\lambda_{k}(M) \sim \operatorname{vol}^{-2}(M)$ up to a multiplicative constant depending only on $\varepsilon, c$, and $k$, where $\lambda_{k}(M)$ is the $k$ th eigenvalue of the Laplace-Beltrami operator.

\section{Introduction}

Recall that the Laplace-Beltrami operator $\Delta=-\operatorname{div} \nabla$ on a closed Riemannian manifold $M$ is a self-adjoint linear operator defined on $H^{2}(M)$, the Sobolev space of twice weakly differentiable $L^{2}$-integrable functions on $M$. The set of values $\lambda$ satisfying $\Delta f=\lambda f$ is positive, discrete, and unbounded and can be ordered

$$
0=\lambda_{0}<\lambda_{1} \leqslant \lambda_{2} \leqslant \cdots .
$$

The main result of this paper relates the $k$ th eigenvalue value of the Laplace-Beltrami operator of certain closed hyperbolic 3-manifolds to their volume. Biringer and Souto proved in [3] that, given $\varepsilon, c, \delta>0$, there exist only finitely many isometry classes of hyperbolic 3 -manifolds $M$ with $\operatorname{inj}(M) \geqslant \varepsilon, \operatorname{rank}\left(\pi_{1}(M)\right) \leqslant c$, and $\lambda_{1}>\delta$. In light of Wang's Finiteness Theorem for $\varepsilon$ thick hyperbolic 3 -manifolds, this is equivalent to saying that, with injectivity radius and rank bounds in place, $\lambda_{1}(M) \rightarrow 0$ as $\operatorname{vol}(M) \rightarrow \infty$. The following theorem implies their result and additionally provides a precise asymptotic statement.

Theorem 1. For every $\varepsilon>0, c, k \in \mathbb{N}$, there exists $\Omega(\varepsilon, c, k)$ such that, if $M$ is a closed, $\varepsilon$-thick hyperbolic 3-manifold with $\operatorname{rank}\left(\pi_{1}(M)\right)<c$, then

$$
\frac{1}{\Omega \operatorname{vol}^{2}(M)} \leqslant \lambda_{k}(M) \leqslant \frac{\Omega}{\operatorname{vol}^{2}(M)},
$$

where $\lambda_{k}(M)$ is the $k$ th positive eigenvalue of the Laplace operator on $M$.

Recall that the rank of a group is the minimal number of elements in a generating set. The injectivity radius of a closed hyperbolic manifold $M$ is half the length of the smallest essential closed curve in $M$. If the injectivity radius of a manifold $M$ is greater than $\varepsilon$, then we say that the manifold is $\varepsilon$-thick.

Since we will refer to the hypotheses of Theorem 1 repeatedly, from now on we will say that,

Given $\varepsilon$ and $c$, a closed hyperbolic 3-manifold $M$ satisfies $(*)$ if the following holds:

$$
(*) \quad \operatorname{inj}(M) \geqslant \varepsilon \quad \text { and } \quad \operatorname{rank}\left(\pi_{1}(M)\right) \leqslant c
$$


This notation represses $\varepsilon$ and $c$, but will always be used in the context of fixed $\varepsilon$ and $c$. Note that for suitable choices of $\varepsilon$ and $c$, there are infinitely many examples of manifolds satisfying the $(*)$; think, for instance, of cyclic covers of a manifold fibering over the circle.

We discuss briefly the role of the various constants in the hypotheses of the theorem. The lower bound in the theorem was proved by Schoen [15] and depends neither on $k, c$, nor $\varepsilon$. Further, Schoen's result holds more generally for Riemannian 3-manifolds with pinched negative sectional curvature. The upper bound, however, definitively depends on $c$ : LongLubotzky-Reid showed in [13] that every closed hyperbolic 3-manifold has a co-final family of covers $\left\{M_{i}\right\}$ with Property $\tau$. For any such family, $\operatorname{vol}\left(M_{i}\right) \rightarrow \infty$ and $\lim \inf \lambda_{1}\left(M_{i}\right)>0$. The upper bound also definitively depends on $k$ because $\lambda_{k}(M) \rightarrow \infty$ as $k \rightarrow \infty$. The proof of the upper bound we present certainly depends on the injectivity radius bound $\varepsilon$, and the author highly suspects that it is necessary for the theorem to hold. Lastly, the proof given also heavily relies on the structure provided by hyperbolicity. In general, some kind of curvature bound is necessary because Colbois and Dodziuk proved that every compact manifold $M^{n}$ with $n \geqslant 3$ admits metrics $g$ of volume 1 with arbitrarily large $\lambda_{1}\left(M_{g}\right)$ (see $\left.[\mathbf{9}]\right)$. See $[\mathbf{5}, \mathbf{7}, \mathbf{1 0}]$ for similar upper and lower bounds on $\lambda_{1}(M)$ in terms of volume in the case that $M$ is non-compact.

To prove the upper bound in Theorem 1, we use the 'max' part of the Minimax theorem. Loosely speaking, we can bound $\lambda_{k}$ from above by constructing test functions with bounded Rayleigh quotient. With the end of constructing these functions, we discuss a theorem of Biringer and Souto that gives us (1) a topological decomposition of our manifold into pieces including large-diameter product regions, and (2) a point-wise geometric statement about these product regions. We strengthen their geometric statement, showing that these product regions can be fibered by surfaces of bounded geometry. Finally, using this fact, construct step-like test functions with bounded Rayleigh quotients.

\section{Preliminaries}

We recall some well-known facts and definitions about the Laplacian, hyperbolic geometry, and 3-manifold topology. For more in-depth treatment of these subjects see, for example, $[1,6,11,14,16]$.

\subsection{Minimax theorem}

To bound $\lambda_{k}$ from above, we use the 'max' half of the Minimax theorem, namely:

Minimax theorem. Let $h_{0}, \ldots, h_{k} \in C^{\infty}(M)$ be positive functions satisfying

$$
\tau \operatorname{vol}\left(\operatorname{supp} h_{i} \cap \operatorname{supp} h_{j}\right)=0,
$$

for every $0 \leqslant i<j \leqslant k$. Then

$$
\lambda_{k}(M) \leqslant \max _{0 \leqslant j \leqslant k} R\left(h_{j}\right)
$$

where $R\left(h_{j}\right)$ is the Rayleigh quotient:

$$
R\left(h_{j}\right)=\frac{\int_{M}\left\|\nabla h_{j}\right\|^{2} d M}{\int_{M} h_{j}^{2} d M} .
$$

See $[6]$ for a proof.

Using the Rayleigh quotient is a common tool for bounding eigenvalues of the Laplacian. See, for example, Canary's result on bounds for $\lambda_{0}$ for infinite-volume hyperbolic manifolds [7], Cheeger's inequality [8], and Huber's result on $\lambda_{1}$ for Riemann surfaces [12]. 


\subsection{Geometric convergence}

To prove Theorem 1, we will often work with geometrically convergent sequences of pointed hyperbolic 3-manifolds.

Recall that a sequence of framed manifolds $\left\{\left(M_{i}, \omega_{i}\right)\right\}$ converges geometrically to $(M, \omega)$ if there exist sequences $\left\{R_{i}\right\} \rightarrow \infty$ and $\left\{L_{i}\right\} \rightarrow 1$, such that for each $i$ there is an $L_{i}$-bi-Lipschitz embedding $N_{R_{i}}(x) \rightarrow M_{i}$ taking $\omega \mapsto \omega_{i}$, where $N_{R_{i}}\left(x_{i}\right)$ is the $R_{i}$-neighborhood of $x_{i}, \omega_{i}$ an orthonormal frame for $T_{x_{i}} M_{i}$. This is sometimes called Gromov-Hausdorff convergence. We often repress $\left\{R_{i}\right\}$ and $\left\{L_{i}\right\}$ and describe this colloquially by saying that the $M_{i}$ approximate $M$ for large enough $i$. (Recall that this is equivalent to convergence of the groups of covering transformations with respect to the Chabauty topology.)

In many cases, it will be sufficient to forget about the baseframe and talk about convergence of pointed manifolds (this time without baseframe): $\left\{\left(M_{i}, x_{i}\right)\right\}, x_{i} \in M_{i}$.

When we have lower bounds on injectivity radius, we have the following useful theorem.

Proposition 2 (see [1] for a proof). If a sequence of pointed hyperbolic 3-manifolds $\left(M_{i}, x_{i}\right)$ has a positive lower bound on the injectivity radii at the basepoints $x_{i}$, then some subsequence converges geometrically.

This means that as long as basepoints are chosen in the thick part, sequences will always converge up to subsequence.

A consequence of the above proposition and Mostow's Rigidity Theorem is Wang's finiteness theorem. In three dimensions, the theorem is the following.

Theorem 3 (Wang's finiteness theorem for dimension $3[\mathbf{1 7}]$ ). Given $\varepsilon, V>0$, there exist only finitely many closed hyperbolic 3 -manifolds with injectivity radius greater than $\varepsilon$ and volume less than $V$.

This will be important in the proof of Theorem 1 because it allows us to restrict our attention to large-volume manifolds. This is also why Theorem 1 implies Biringer and Souto's finiteness theorem $[\mathbf{3}]$.

There is another notion of geometric convergence, smooth geometric convergence, in which we require the embeddings $N_{R_{i}}(x) \rightarrow M_{i}$ to be smooth and to converge smoothly to an isometric embedding. Although this sounds a priori stronger than Gromov-Hausdorff convergence, they are actually equivalent. See [1, Theorem E.1.13] for a precise definition of smooth convergence and for the proof that it is equivalent to the Chabauty topology.

\subsection{Doubly degenerate manifolds}

An important lemma in the proof of Theorem 1 describes sequences of finite-volume hyperbolic 3-manifolds whose geometric limits are doubly degenerate. Recall that a doubly degenerate manifold (of genus $g$ ) is a hyperbolic 3-manifold without parabolics homeomorphic to $\Sigma_{g} \times \mathbb{R}$ with two simply degenerate ends, where $\Sigma_{g}$ is a closed surface of genus $g$. Recall that, in the absence of cusps, an end of a hyperbolic 3-manifold is simply degenerate if there exists a sequence of essential simple closed curves exiting the end. See [4] for a detailed discussion of ends of hyperbolic 3-manifolds.

See $[2]$ for a sketch of the proof of the following well-known fact. 
FACT 4. Let $\mathcal{M}_{g, \varepsilon}$ be the set of $\varepsilon$-thick, doubly degenerate pointed manifolds homeomorphic to $\Sigma_{g} \times \mathbb{R}$, where $\Sigma_{g}$ is a closed surface of genus $g$. Then $\mathcal{M}_{g, \varepsilon}$ is compact with respect to geometric convergence.

\subsection{Product regions}

Lastly, we discuss a few topological facts we will need about product regions. These definitions and facts will certainly be known to the expert, but we include them here for completeness.

Recall that an open (respectively, closed) product region is a 3-manifold homeomorphic to $\Sigma \times(0,1)$ (respectively, $\Sigma \times[0,1]$ ), where $\Sigma$ is a closed orientable surface.

Any embedded surface in a product region homotopic to a boundary component will be called a fiber. We say that an embedded surface in a product region is separating if its complement is disconnected. A separating surface either bounds a compact region not meeting the boundary, or it separates the boundary components. That is, the boundary components lie in distinct components of its complement. Equivalently, any arc connecting the boundary components necessarily meets the surface.

If a compact 3-manifold $M$ has boundary $\partial M$ such that $\partial M$ contains no 2-spheres, but does contain a compact connected surface $F$ such that the canonical inclusion $i_{*}: \pi_{1}(F) \rightarrow \pi_{1}(M)$ is an isomorphism, then $M$ is a product region [11, 10.2]. Therefore, given a product region $M$, a boundary component of $M$ and any embedded fiber of $M$ not touching that boundary component together bound a product region. Iterating this fact gives us a useful statement we will use repeatedly.

FACT 5. Two disjoint embedded fibers in a product region themselves bound a product region.

Now, let $T=\Sigma \times I$ be a product region of genus $g$. Let $\Sigma^{\prime}$ be a closed embedded surface in $T$. We can view $\Sigma^{\prime}$ as an element of $H_{2}(T ; \mathbb{Q})$. Recall that there exists a non-degenerate bilinear pairing $H_{1}(T, \partial T ; \mathbb{Q}) \times H_{2}(T ; \mathbb{Q})$ given by linear extension of intersection number. Because $\operatorname{dim} H_{1}(T, \partial T ; \mathbb{Q})=\operatorname{dim} H_{2}(T ; \mathbb{Q})=1$, the following dichotomy holds: either $\Sigma^{\prime}$ has non-zero algebraic intersection number with the arc $\{x\} \times[0,1]$, for some $x \in \Sigma$, in which case it separates the boundary components of $T$, or $\Sigma$ is null-homologous, in which case it bounds a compact region not meeting the boundary. In particular, we have the following fact.

FACT 6. There are no embedded, non-separating surfaces in $T$.

Continuing with the same notation, let $p$ be the projection:

$$
p: T=\Sigma \times I \longrightarrow \Sigma \text { given by }(x, t) \longmapsto x .
$$

If $\Sigma^{\prime}$ has genus less than $g$, then the restriction $\left.p\right|_{\Sigma^{\prime}}$ cannot be $\pi_{1}$-injective. In other words, $\Sigma^{\prime}$ is compressible in $T$ (see [11]) and by repeatedly compressing, we reduce genus and end up with a sphere, which must be null-homologous because the universal cover of $T$ is contractible. Being null-homologous, $\Sigma^{\prime}$ bounds a compact region not meeting the boundary. In summary, we have the following fact.

FACT 7. If $\Sigma^{\prime}$ is a closed embedded orientable surface in $T$ with genus smaller than $g$, then $\Sigma^{\prime}$ bounds a compact region. Conversely, if $\Sigma^{\prime}$ separates the boundary components of $T$, then the genus of $\Sigma^{\prime}$ is greater than or equal to that of $T$. 
If $\Sigma^{\prime}$ is an embedded surface of genus equal to $g$ (the genus of the product region) and separates the boundary components of $T$, then $\Sigma^{\prime}$ is non-trivial in $H_{2}(T ; \mathbb{Q})$. As such, $\left.p\right|_{\Sigma^{\prime}}$ must be $\pi_{1}$-injective, otherwise $\Sigma^{\prime}$ would be homologous to a smaller-genus surface and therefore null-homologous by Fact 7. Because $\left.p\right|_{\Sigma^{\prime}}$ is $\pi_{1}$-injective, it is homotopic to a covering map and, since the genera are the same, homotopic to a homeomorphism. In other words, we have the following fact.

FACT 8. If $\Sigma^{\prime}$ and $T$ have the same genus and $\Sigma^{\prime}$ separates the boundary components of $T$, then $\Sigma^{\prime}$ is a fiber in $T$.

\section{A decomposition theorem}

The proof of Theorem 1 follows quite easily once we establish a useful decomposition theorem for manifolds satisfying $(*)$. We start by introducing a theorem of Biringer and Souto which gives us a decomposition of $M$ satisfying $(*)$ into pieces including large-diameter product regions. The bulk of the work in this chapter is proving a stronger version of their theorem, Theorem 10, which shows that those product regions can be fibered by surfaces of bounded geometry. From there, the decomposition theorem, Corollary 15, follows immediately.

\subsection{Biringer-Souto theorem}

In their forthcoming paper, Biringer and Souto ('Thick 3-manifolds with bounded rank') proved the following theorem:

Theorem 9 (Biringer-Souto). For every $\varepsilon, c>0$, there exists a finite set $\left\{Q_{1}, \ldots, Q_{s}\right\}$ of compact Riemannian 3-manifolds (perhaps with boundary) and $L, r>1$ such that if $M$ is a closed hyperbolic 3-manifold with

$$
(*) \quad \operatorname{inj}(M) \geqslant \varepsilon \text { and } \operatorname{rank}\left(\pi_{1}(M)\right) \leqslant c,
$$

then $M$ contains a compact submanifold $\bar{M}$ with the following properties.

(1) The compact submanifold $\bar{M}$ has at most $r$ components, each one of them L-bi-Lipschitz equivalent to one of the $Q_{i}$.

(2) Each component of $M-\bar{M}$ is homeomorphic to $\Sigma_{g} \times \mathbb{R}$, where $\Sigma_{g}$ is a closed orientable surface of some genus $g$.

Further, given any pairwise distinct pointed sequence $\left(M_{i}, x_{i}\right)$ such that each $M_{i}$ satisfies $(*)$ and $d\left(x_{i}, \overline{M_{i}}\right) \rightarrow \infty$, some subsequence converges geometrically to $\left(M_{\infty}, x_{\infty}\right)$, where $M_{\infty}$ is an $\varepsilon$-thick doubly degenerate manifold.

We refer to $\bar{M}$ as the tiny manifold because the volume of $\bar{M}$ is bounded above by a constant depending only on $\varepsilon$ and $c$. The first part of the theorem tells us that components of the complement of the tiny manifold are all product regions, a purely topological statement. A helpful picture to illustrate the theorem is shown in Figure 1.

Let us point out what we do not know immediately from Theorem 9. The first part of the theorem is purely topological, and does not give geometric information about the product regions. The second part of the theorem is geometric, but is only a pointwise statement. A neighborhood of a point far enough away from the tiny manifold will look like a product region neighborhood in a doubly degenerate manifold of genus $g$, but a priori this local structure may 


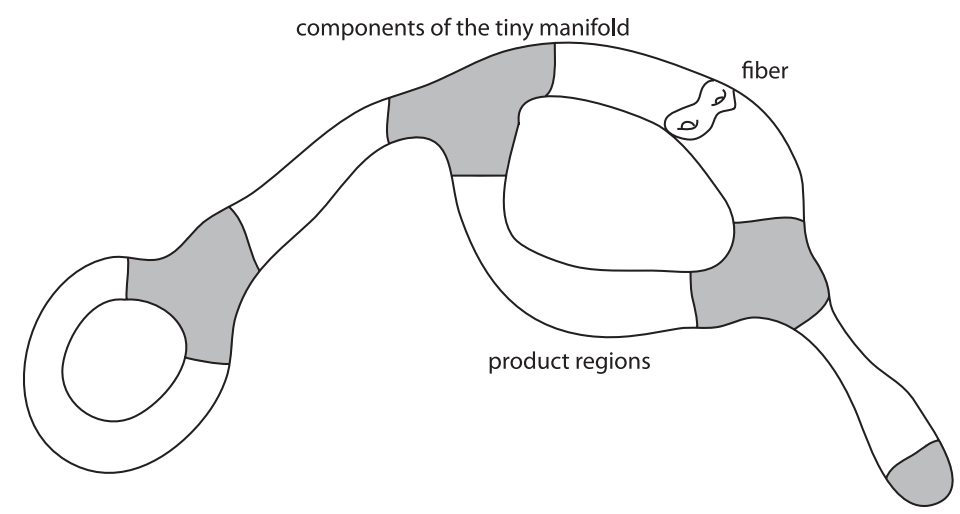

Figure 1. A picture illustrating the Biringer-Souto theorem. The 'tiny manifold' $\bar{M}$ is shown in gray. The white parts are product regions.

not be compatible with the topological structure of the larger product region component, in particular, a priori it may not be the same genus.

\subsection{A stronger version}

We improve this loose point-wise geometric statement by showing that we can actually fiber these product regions with surfaces of uniformly bounded geometry. To this end, we frequently refer to a fixed metric family of 'canonical' product regions $\left\{S_{g} \times[-1,1] \mid S_{g} \in \mathcal{S}\right\}$ endowed with the product metric, where $\mathcal{S}=\left\{S_{g}\right\}_{g=2}^{\infty}$ is a fixed family of hyperbolic structures, one for each genus $g \geqslant 2$.

The main result of this section is the following theorem.

TheOrem 10. For every $\varepsilon, c>0$, there exists a finite set $\left\{Q_{1}, \ldots, Q_{s}\right\}$ of compact Riemannian 3-manifolds (perhaps with boundary) and $L, r, G>1$ such that if $M$ is a closed hyperbolic 3-manifold with

$$
(*) \operatorname{inj}(M) \geqslant \varepsilon \text { and } \operatorname{rank} \pi_{1}(M) \leqslant c,
$$

then $M$ contains a compact submanifold $\widetilde{M}$ with the following properties.

(1) The compact submanifold $\widetilde{M}$ has at most $r$ components, each one of them L-bi-Lipschitz equivalent to one of the $Q_{i}$.

(2) Each component $T$ of $M-\widetilde{M}$ is homeomorphic to $\Sigma_{g} \times \mathbb{R}$, where $\Sigma_{g}$ is a closed orientable surface of genus $g<G$.

(3) Further, there is a foliation of $T$ by genus- $g$ surfaces compatible with the product structure such that for any point $x \in T$, there is a leaf-preserving $L$-bi-Lipschitz embedding

$$
S_{g} \times[-1,1] \longrightarrow M, \quad S_{g} \in \mathcal{S},
$$

with $x$ in the image of the 0-fiber.

\subsection{Proof of Theorem 10}

We start by showing the existence of $G$ in the statement of Theorem 10 . 


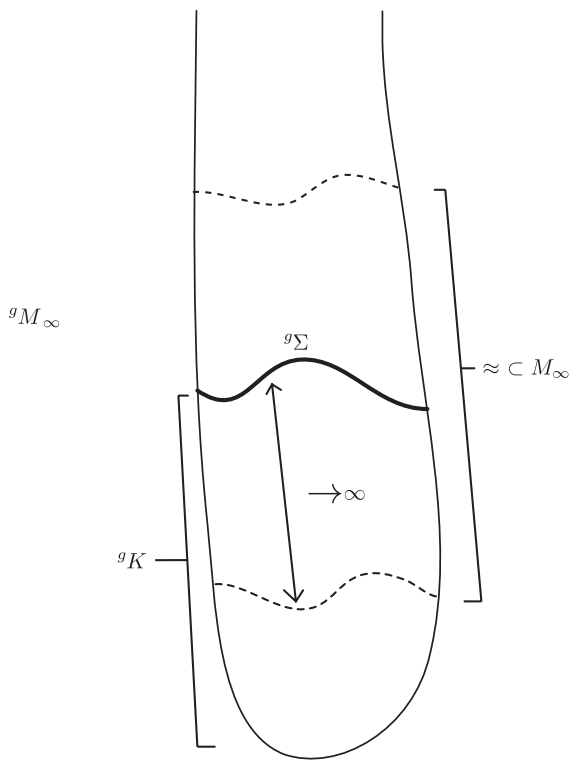

Figure 2. As $g \rightarrow \infty$, the diameter of ${ }^{g} K$ must also go to $\infty$.

Lemma 11. There exists $G$ such that given $M_{i}$ satisfying $(*)$ and $x_{i} \in M_{i}$ with $d\left(\overline{M_{i}}, x_{i}\right) \rightarrow$ $\infty$, then the doubly degenerate geometric limit $M_{\infty}$ guaranteed by Theorem 9 has genus at most $G$.

Proof. Suppose that no such bound on genus existed. Then for infinitely many $g \in \mathbb{N}$, there exists a sequence of pointed manifolds $\left({ }^{g} M_{i},{ }^{g} x_{i}\right)$ satisfying $(*)$ with $d\left(\overline{g^{g} M_{i}},{ }^{g} x\right) \rightarrow \infty$ and converging to a doubly degenerate manifold $\left({ }^{g} M_{\infty},{ }^{g} x_{\infty}\right)$ of genus $g$. Because each ${ }^{g} M_{\infty}$ is $\varepsilon$-thick, we can assume that up to subsequence $\left({ }^{g} M_{\infty},{ }^{g} x_{\infty}\right)$ converges to some $\left(M_{\infty}, x_{\infty}\right)$. Note that $\left(M_{\infty}, x_{\infty}\right)$ is also the geometric limit of some diagonal sequence $\left({ }^{g} M_{i},{ }^{g} x_{i}\right)$, and, as such, is an $\varepsilon$-thick doubly degenerate manifold of some genus $G$ by Theorem 9 .

Fix an embedded fiber $\Sigma \subset M_{\infty}$ through $x_{\infty}$. For $g>G$ large enough, we have a 2-bi-Lipschitz map from a domain containing $\Sigma \subset M_{\infty}$ to ${ }^{g} M_{\infty}$. From now on, we will describe this situation by saying 'for $g$ large enough we can approximate $\Sigma \subset M_{\infty}$ by ${ }^{g} \Sigma \subset \subset^{g} M_{\infty}$ '. Because ${ }^{g} \Sigma$ has genus $G$ and ${ }^{g} M_{\infty}$ has genus $g>G,{ }^{g} \Sigma$ bounds a compact region ${ }^{g} K \subset{ }^{g} M_{\infty}$ by Fact 7 . Note that $\operatorname{diam}\left({ }^{g} K\right) \rightarrow \infty$ because otherwise ${ }^{g} K$ would lie entirely in a local approximation of $M_{\infty}$, which is impossible; see Figure 2.

Similarly, for each $g$, we can choose index $i(g)$ large enough to approximate ${ }^{g} K \subset{ }^{g} M_{\infty}$ by ${ }^{g} K_{i(g)} \subset{ }^{g} M_{i(g)}$. Note also that $\operatorname{diam}\left({ }^{g} K_{i(g)}\right) \rightarrow \infty$.

We are now ready to shed this elaborate notation: rename the sequence $\left({ }^{g} M_{i(g)},{ }^{g} x_{i(g)}\right)$ with one index $j$. Likewise, let $K_{j}={ }^{g} K_{i(g)}$ and let $\Sigma_{j}=\partial K_{j}$.

Let $x_{j}^{\prime} \in K_{j}$ be a point furthest from $\Sigma_{j}$. Note that $d\left(\overline{M_{j}}, x_{j}^{\prime}\right) \rightarrow \infty$ so Theorem 9 applies and up to subsequence we have limit $\left(M_{j}, x_{j}^{\prime}\right) \rightarrow\left(M_{\infty}^{\prime}, x_{\infty}^{\prime}\right)$, where $M_{\infty}^{\prime}$ is doubly degenerate of some genus $G^{\prime}$.

Fix an embedded fiber $\Sigma^{\prime} \subset M_{\infty}^{\prime}$ through $x_{\infty}^{\prime}$. Also fix an infinite arc $\gamma: \mathbb{R} \rightarrow M_{\infty}^{\prime}$ exiting both ends. Note that $\gamma$ necessarily has algebraic intersection number 1 with $\Sigma^{\prime}$.

Let $\Sigma_{j}^{\prime} \subset M_{j}$ be the approximation of $\Sigma^{\prime}$ that exists for large enough $j$. We briefly outline the rest of the proof: working in the approximations $M_{j}$, we use approximations of $\gamma$ to find 


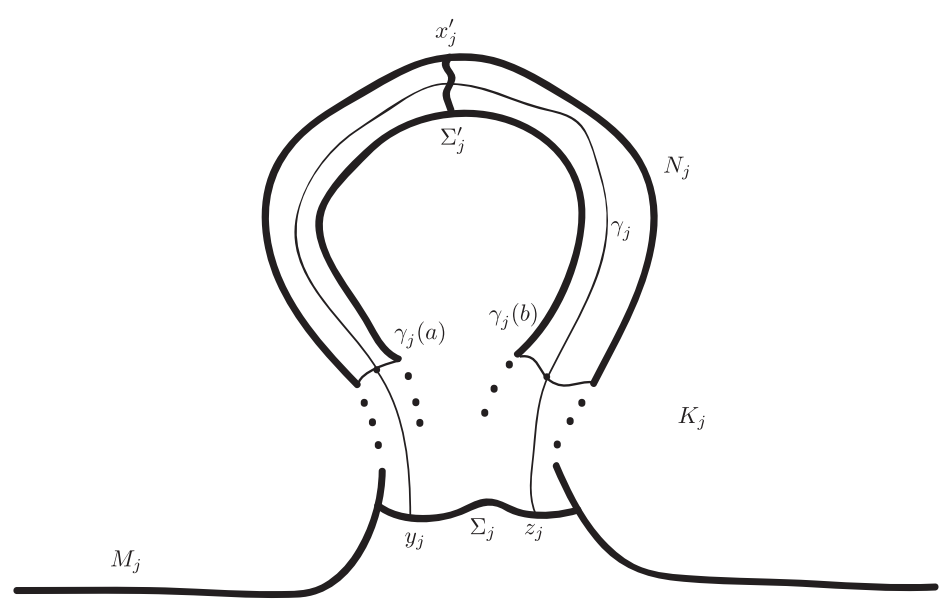

FIGURE 3. The closed surface $\Sigma_{j}^{\prime}$ is an embedded non-separating surface, a contradiction.

closed curves intersecting $\Sigma_{j}^{\prime}$ exactly once, a contradiction, since $\Sigma_{j}^{\prime}$ must have disconnected complement by Fact 6 .

The closed curves will come from concatenating the $\gamma$-approximations with geodesic segments connecting to $\Sigma_{j}=\partial K_{j}$, such segments cannot backtrack and go back through $\Sigma_{j}^{\prime}$ because the regions of approximation have very large diameters and $x_{j}^{\prime}$ is the furthest point from $\Sigma_{j}=\partial K_{j}$.

To help with the following nitty-gritty details, see Figure 3 . We have increasingly large approximations $N_{j} \subset M_{j}$ of $M_{\infty}^{\prime}$. Because $\operatorname{diam}\left(K_{j}\right) \rightarrow \infty$, large enough $j$ allow us to impose the simultaneous restrictions: $\operatorname{diam}\left(N_{j}\right) \gg \operatorname{diam}\left(\Sigma_{j}^{\prime}\right)$ and $N_{j} \subset K_{j}$.

Note that $N_{j}$ may have more than two boundary components, but that the boundary components fall into two classes depending on which component of $N_{j}-\Sigma_{j}^{\prime}$ they belong to. The distance between these two classes of boundary components is at least $d_{j}$, where

$$
d_{j}=\operatorname{diam}\left(N_{j}\right) / 2-\operatorname{diam}\left(\Sigma_{j}^{\prime}\right),
$$

and thus $\lim _{j \rightarrow \infty} d_{j}=\infty$.

We are now ready to construct our closed curve intersecting $\Sigma_{j}^{\prime}$ exactly once. Let $\gamma_{j}:[a, b] \rightarrow$ $N_{j}$ be the unique component of the approximation of $\gamma$ with image intersecting $\Sigma_{j}^{\prime}$. (We need to specify a particular component because we may have other small segments near $\partial N_{j}$.) Concatenate $\gamma_{j}$ with geodesics segments $\left[\gamma_{j}(a), y_{j}\right],\left[\gamma_{j}(b), z_{j}\right]$, such that $y_{j}, z_{j} \in \Sigma_{j}$ are the points closest to $\gamma_{j}(a)$ and $\gamma_{j}(b)$, respectively, recalling that $\Sigma_{j}=\partial K_{j}$. Finally, concatenate with any arc in $\Sigma_{j}$ connecting $y_{j}$ to $z_{j}$. See Figure 3 for an illustration. We claim that this closed curve still intersects $\Sigma_{j}^{\prime}$ exactly once, showing that the complement to $\Sigma_{j}^{\prime}$ is connected, contradicting Fact 6 .

We have only to show that $\left[\gamma_{j}(a), y_{j}\right]$ and $\left[\gamma_{j}(b), z_{j}\right]$ do not intersect $\Sigma_{j}^{\prime}$. This is easy, for recall that $d_{j} \gg \operatorname{diam}\left(\Sigma_{j}^{\prime}\right)$. Without loss of generality, suppose that $\left[\gamma(a), y_{j}\right]$ intersected $\Sigma_{j}^{\prime}$, then by the triangle inequality $d\left(\gamma_{j}(a), \Sigma_{j}\right)>d\left(x_{j}^{\prime}, \Sigma_{j}\right)$, contradicting our initial choice of $x_{j}^{\prime}$.

From now on, let $G$ be the constant provided by Lemma 11 .

Note that in the course of the above proof, we showed the following fact. 
FACT 12. If a sequence of metric product regions $\left(T_{i}, x_{i}\right)$ converge geometrically to a doubly degenerate manifold $\left(M_{\infty}, x_{\infty}\right)$, then given a fiber of $M_{\infty}$ through $x_{\infty}$, the approximating image of the fiber in $T_{i}$ separates the boundary components of $T_{i}$ for all sufficiently large $i$.

Recall that the space of $\varepsilon$-thick doubly degenerate manifolds of genus $g$ is compact with respect to geometric convergence (cf. Fact 4). It follows that the space of $\varepsilon$-thick doubly degenerate manifolds of genus $g \leqslant G$ is also compact with respect to geometric convergence. Recall that we fixed a family of hyperbolic surfaces $\mathcal{S}=\left\{S_{g}\right\}_{g=2}^{\infty}$. Compactness yields uniformity in the following sense:

(DD1) Given $\varepsilon>0, G \geqslant 2$, there exists $L=L(\varepsilon, G)$ such that if $E$ is $\varepsilon$-thick and doubly degenerate of genus $g \leqslant G$, and $x \in E$, then there exists an $L$-bi-Lipschitz embedding $\varphi: S_{g} \times[-1,1] \rightarrow E, S_{g} \in \mathcal{S}$, such that $x \in \varphi\left(S_{g} \times\{0\}\right)$.

The next statement follows easily from the preceding statement by letting

$$
D(\varepsilon, G)=L(\varepsilon, G) \max _{2 \leqslant g \leqslant G}\left\{\operatorname{diam}\left(S_{g}\right) \mid S_{g} \in \mathcal{S}\right\},
$$

but we will frequently need this specific formulation.

(DD2) Given $\varepsilon>0, G \geqslant 2$, there exists $D=D(\varepsilon, G)$ such that if $E$ is $\varepsilon$-thick and doubly degenerate of genus $g \leqslant G$, and $x \in E$, then there is an embedded fiber through $x$ of diameter less than $D$.

If $E$ is $\varepsilon$-thick and doubly degenerate of genus $g \leqslant G$, then (DD2), Fact 5 , and the triangle inequality combine to give the following obvious (but useful) statement:

(DD3) Let $B$ be a ball of radius $R$ in $E$. Then $B$ contains a product region of width $w \geqslant$ $2 R-4 D(\varepsilon, G)$. That is, large-radius balls in $E$ contain comparably large-width product regions.

Recall that the width of a product region is the shortest distance between its boundary components.

We now give a slightly refined version of the geometric content of Theorem 9 incorporating the genus bound we just proved.

Corollary 13. For every $\varepsilon, c>0, R, L>1$, there exists $\delta(\varepsilon, c, R, L)$ such that if $M$ satisfies $(*)$ and $x \in M$ such that $d(x, \bar{M})>\delta$, then there is a product region $T_{x} \subset M-\bar{M}$ such that

(i) $N_{R}(x) \subset T_{x}$;

(ii) there exists an L-bi-Lipschitz embedding $f_{x}: T_{x} \rightarrow E$, where $E$ is doubly degenerate of genus $g \leqslant G(\varepsilon, c)$, such that $f$ is homotopic to a fiber in $E$;

(iii) $T_{x}$ separates the boundary components of $P_{x}$, where $P_{x}$ is the product-region component of $M-\bar{M}$ containing $x$.

Proof. Suppose that no such $\delta$ exists. Then there exists a sequence of closed hyperbolic 3-manifolds $\left(M_{i}, x_{i}\right)$ satisfying $(*)$ with $d\left(\overline{M_{i}}, x_{i}\right) \rightarrow \infty$ such that for all $i$ no such $f_{x_{i}}$ approximation exists. However, by Theorem 9 , there is doubly degenerate manifold $M_{\infty}$ such that $\left(M_{i}, x_{i}\right) \rightarrow\left(M_{\infty}, x_{\infty}\right)$. By the definition of geometric convergence, there exist $L_{i} \rightarrow 1$ and $R_{i} \rightarrow \infty$ and $L_{i}$-bi-Lipschitz maps $g_{x_{i}}:\left(N_{R_{i}}\left(x_{\infty}\right), x_{\infty}\right) \rightarrow\left(M_{i}, x_{i}\right)$. By (DD3), we can restrict the inverse to comparably large-width product regions $T_{x_{i}} \subset M_{i}$ and get maps $f_{x_{i}}: T_{x_{i}} \rightarrow M_{\infty}$. 
For large enough $i$, these approximations satisfy the hypotheses of the theorem, contradicting what we assumed.

By Fact 12 , the $T_{x_{i}}$ eventually separate, showing (iii).

At this point, we would like to improve Corollary 13 in two ways. First, the above corollary describes only local behavior; we would like to have compatibility of the embeddings described above. That is, we want to know that the embeddings $T_{x} \rightarrow E$ and $T_{x^{\prime}} \rightarrow E$ are homotopic for different points $x$ and $x^{\prime}$ in the same component of $M-\bar{M}$ (far enough away from $\bar{M}$ ). Second, we would like to compare the product regions $T_{x}$ with concrete product regions instead of some doubly degenerate manifold $E$.

Recall that we fixed a family of hyperbolic surfaces $\mathcal{S}=\left\{S_{g}\right\}_{g=2}^{\infty}$ and associated product regions $\left\{S_{g} \times[-1,1] \mid S_{g} \in \mathcal{S}\right\}$ endowed with the product metric. We say that a metric product region $T$ in an ambient manifold $M$ is locally fibered by genus- $g$ surfaces of $L$-bounded geometry if for every $x \in T$ there exists an $L$-bi-Lipschitz embedding $g_{x}: S_{g} \times[-1,1] \rightarrow M$ homotopic to a fiber in $T$ such that $x$ lies in the image of the 0 -fiber.

Lemma 14. Given $\varepsilon$ and $c$, let $M$ satisfy $(*)$. Then there exists $w, G, \delta>0, L>1$ such that, given a component $P \subset M-\bar{M}$ of width greater than $w$, there exists a submanifold $T \subset P$ with $P \subset N_{\delta}(T)$ such that $T$ is a product region of some genus $g \leqslant G$ and $T$ is locally fibered by genus- $g$ surfaces of $L$-bounded geometry.

That is, all but a boundedly finite neighborhood of $\partial P$ is locally fibered by surfaces of bounded geometry.

Proof. Let $G$ be as in Lemma 11. Roughly speaking, we choose a finite set of points $\left\{x_{i}\right\} \subset P$ far enough away from $\partial P$ to use Corollary 13 and find associated large-diameter product regions $T_{x_{i}}$ with union $T=\bigcup_{i} T_{x_{i}} \subset P$. We then use topological facts to show that the $T_{x_{i}}$ are all homotopic and their union $T$ is a product region. Lastly, we show that $T$ is locally fibered by surfaces of bounded geometry.

Step 1: Consider any component $P \subset M-\bar{M}$ of width $w>2 \delta(\varepsilon, c, R, 1.5)$, where $R=$ $3(L(\varepsilon, G)+D(\varepsilon, G))$ and $\delta=\delta(\varepsilon, c, R, 1.5)$ comes from Corollary 13. Let $\gamma:[0, w] \rightarrow P$ be a geodesic segment parameterized by arc length with endpoints in distinct components of $\partial P$. Let $t_{1}=\delta$ and let $x_{1}=\gamma\left(t_{1}\right)$. Let $T_{x_{1}}$ be a product region associated to $x_{1}$ given by Corollary 13 . For $i \geqslant 2$, define $t_{i}, x_{i}$ and $T_{x_{i}}$ inductively by

$$
\begin{aligned}
& t_{i}=\max _{t \in\left[t_{i-1}, w-\delta\right]}\left\{t \mid \gamma(t) \cap T_{x_{i-1}} \neq \emptyset\right\}, \\
& x_{i}=\gamma\left(t_{i}\right), \\
& T_{i} \text { is a product region associated to } x_{i} \text { given by Corollary } 13 .
\end{aligned}
$$

Let $T=\bigcup_{i} T_{x_{i}}$. Note that the proof of Corollary 13, (DD2), and (DD3) allow us to assume that the diameters of the components of $\partial T_{x_{i}}$ are uniformly bounded above by $1.5 D(\varepsilon, G)$. Thus, by our choice of $R$, the boundary surfaces of all the $T_{x_{i}}$ are pairwise disjoint. Also note that $P \subset N_{\delta+d}(T)$, where $d$ is the maximum possible diameter of any boundary component of the tiny manifold $\partial \bar{N}$ over all $N$ satisfying $(*)$; this number is finite because the components of the tiny manifold come from a finite family of $L$-bi-Lipschitz classes.

Step 2: We claim that $T_{x_{1}} \cup T_{x_{2}}$ is a product region. Note that all fibers in $T_{x_{1}}$ and $T_{x_{2}}$ separate the components of $\partial P$ by Corollary 13 . We do not include the details here, but it is not hard to see that some fiber in $T_{x_{2}}$ separates the components of $\partial T_{x_{1}}$ and some fiber in $T_{x_{2}}$ separates the components of $\partial T_{x_{1}}$. Facts 7 and 8 combine to show that the fibers of $T_{x_{1}}$ and $T_{x_{2}}$ have the same genus and are thus homotopic. By Fact 5 and the easy to see fact that 


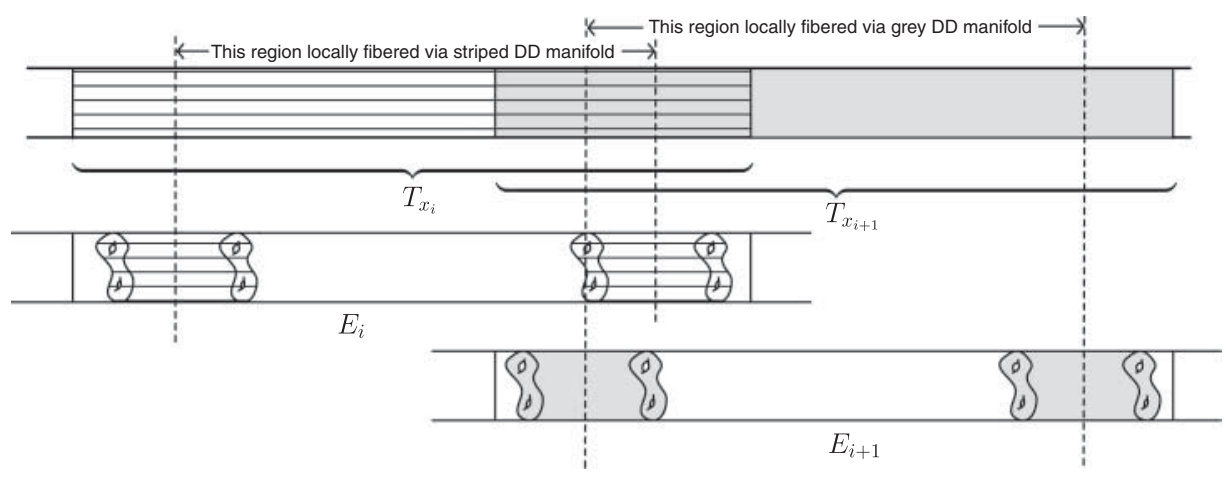

FiguRE 4. The product region $T$ is locally fibered by surfaces of bounded geometry.

the concatenation of product regions is a product region, $T_{x_{1}} \cup T_{x_{2}}$ is a product region. By induction, $T$ is a product region.

Step 3: Note that (DD1) says exactly that doubly degenerate $\varepsilon$-thick manifolds $E$ are locally fibered by surfaces of bounded geometry. Since each $T_{x_{i}}$ looks like a doubly degenerate manifold, we would like to locally fiber $T$ with surfaces of bounded geometry by simply composing the appropriate maps. However, the $T_{x_{i}}$ are bi-Lipschitz equivalent to finite pieces of doubly degenerate $\varepsilon$-thick manifolds, so we have to be a bit careful near the boundary.

Given $i$, let $f_{i}: T_{x_{i}} \rightarrow E_{i}$ be a 1.5-bi-Lipschitz embedding from Corollary 13. Given $x \in T_{x_{i}}$, let $x^{\prime}=f_{i}(x) \in E_{i}$. From (DD1), we have an $L(\varepsilon, G)$-bi-Lipschitz embedding $\varphi_{x^{\prime}}$ : $S_{g} \times[-1,1] \rightarrow E_{i}$ with $x^{\prime}$ in the image of the 0-fiber. In particular, $\varphi_{x^{\prime}}\left(S_{g} \times[-1,1]\right) \subset$ $N_{L(\varepsilon, G)+D(\varepsilon, G)}\left(x^{\prime}\right) \subset E_{i}$. Therefore, if $d\left(x, \partial T_{x_{i}}\right) \geqslant 1.5(L(\varepsilon, G)+D(\varepsilon, G))$, then the image of $\varphi_{x^{\prime}}$ will be contained in the image of $f_{i}$. In particular, $f_{i}^{-1} \circ \varphi_{x^{\prime}}: S_{g} \times[-1,1] \rightarrow T_{x_{i}}$ will be well-defined. Recall that the $T_{x_{i}}$ were defined in such a way to ensure this sufficient overlap. Thus, a desired $1.5 L(\varepsilon, G)$-bi-Lipschitz embedding exists for every point in $T=\bigcup_{i} T_{x_{i}}$. Figure 4 visualizes these overlaps.

REMARK. We have used bi-Lipschitz equivalence in everything we have done so far. Recall that the definition of geometric convergence actually allows us to assume everything we have done is smooth.

We are now ready to prove Theorem 10. We recall the theorem here for reference.

TheOREm 10. For every $\varepsilon, c>0$, there exists a finite set $\left\{Q_{1}, \ldots, Q_{s}\right\}$ of compact Riemannian 3-manifolds (perhaps with boundary) and $L, r, G>1$ such that if $M$ is a closed hyperbolic 3-manifold with

$$
(*) \quad \operatorname{inj}(M) \geqslant \varepsilon \text { and } \operatorname{rank} \pi_{1}(M) \leqslant c,
$$

then $M$ contains a compact submanifold $\widetilde{M}$ with the following properties.

(1) The compact submanifold $\widetilde{M}$ has at most $r$ components, each one of them L-bi-Lipschitz equivalent to one of the $Q_{i}$.

(2) Each component $T$ of $M-\widetilde{M}$ is homeomorphic to $\Sigma_{g} \times \mathbb{R}$, where $\Sigma_{g}$ is a closed orientable surface of genus $g<G$. 
(3) Further, there is a foliation of $T$ by genus- $g$ surfaces compatible with the product structure such that for any point $x \in T$, there is a leaf-preserving $L$-bi-Lipschitz embedding

$$
S_{g} \times[-1,1] \longrightarrow M, \quad S_{g} \in \mathcal{S},
$$

with $x$ in the image of the 0 -fiber.

Proof. To show part (3) of the statement, it is sufficient to show the existence of a finite number of such leaf-preserving embeddings whose images cover $T$ and intersect only on boundary fibers. (Concatenating parts of such parts gives the general result.) We do this by first showing that a 'patchy' foliation exists using Lemma 14. Then we use limits to fill in missing pieces. Lastly, we change our choice of tiny manifold while keeping the components in a finite family of $L$-bi-Lipschitz classes.

Step 1: Let $w$ and $L$ be as in Lemma 14, let $P$ be any component of $M$ of width larger than $w$, and let $T \subset P$ be a genus- $g$ product region as in Lemma 14. (It is possible that no such $P$ exists, in which case, skip to Step 3.) We will cover $T$ with a 'patchy' foliation. That is, we will find a family of $L$-bi-Lipschitz embeddings as in Lemma 14 such that the distance between the images of subsequent maps is bounded both above and below.

Let $w^{\prime}$ be the width of $T$ and let $\gamma:\left[0, w^{\prime}\right] \rightarrow T$ be a geodesic connecting the boundary components of $T$ parameterized by arc length. Let

$$
d=\max \left\{d(x, y) \mid x \in S_{g} \times\{-1\}, y \in S_{g} \times\{1\}\right\},
$$

that is, $d$ is the greatest distance between points in opposite components of $S_{g} \times[-1,1]$.

Let $x_{i}=\gamma(i(L d+1))$ for $i=0,1, \ldots,\left\lfloor w^{\prime} /(L d+1)\right\rfloor$. By Lemma 14, for each $i$ we have an $L$-bi-Lipschitz embedding $f_{i}: S_{g} \times[-1,1] \rightarrow M$ such that $x_{i}$ is in the image of the 0 -fiber. Note that we have spaced the $x_{i}$ so that the distance between the image of $f_{i}$ and $f_{i+1}$ is at least 1 and at most $2 L d+1$. By Fact 5, the remaining components of $T$ not in the image of the $f_{i}$ are also product regions.

Step 2: Our goal now is to complete the patchy foliation above. Given subsequent maps $f_{i}$ and $f_{i+1}$ from Step 1, we fill in the gap by finding an embedding $g: S_{g} \times[-1,1] \rightarrow T$ such that

(i) $g$ is homotopic to $f_{i}$;

(ii) $\operatorname{im} g \cup \operatorname{im} f_{i} \cup \operatorname{im} f_{i+1}$ is a product region;

(iii) $\operatorname{vol}\left(\operatorname{img} g \cap \operatorname{im} f_{i}\right)=\operatorname{vol}\left(\operatorname{im} g \cap \operatorname{im} f_{i+1}\right)=0$.

Given a particular gap, there exists some $L^{\prime}$ such that we can fill in the gap with an $L^{\prime}$-bi-Lipschitz embedding $S_{g} \times[-1,1] \rightarrow T$. At the cost of loosing the 'extremities' of $T$, suppose that there is no universal choice of $\delta^{\prime}, L^{\prime}$ such that, when distance $\delta^{\prime}$ away from $\partial T$ we can fill in gaps with $L^{\prime}$-bi-Lipschitz embeddings. Then there exists a sequence $\left(M_{j}\right)$, with associated $T_{j} \subset P_{j}$ from Lemma 14 with

(i) a gap distance $\delta_{j}^{\prime}$ from $\partial T_{j}$ such that:

(ii) $L_{j}^{\prime}$ is the smallest bi-Lipschitz constant to fill that gap, and

(iii) $L_{j}^{\prime}, \delta_{j}^{\prime} \rightarrow \infty$.

Let $x_{j}$ be any point in the gap in question and consider the limit $\left(M_{j}, x_{j}\right) \rightarrow\left(M_{\infty}, x_{\infty}\right)$ guaranteed by Theorem 9 . The gap in the limit can be filled in with an $L$-bi-Lipschitz map $g: S_{g} \times[-, 1,1] \rightarrow M_{\infty}$ for some $L$. When composed with the approximating maps, this gives us a contradiction.

In summary, given a large-enough-width product region component $P$, there is a subset $T$, which, up to bounded-diameter neighborhoods of the boundary components, can be foliated 
in the desired way. We will abuse notation and now use $T$ to denote that part that can be foliated.

Step 3 : In what follows, we will use $\bar{M}$ to refer to the tiny manifold from the statement of Theorem 9 and $\widetilde{M}$ for our prospective tiny manifold.

We now throw out the parts of $P$ we were not able to foliate and tack them on to the tiny manifold. That is, given $M$ satisfying $(*)$, let $A$ index the finite set $\left\{P_{\alpha}\right\}_{\alpha \in A}$ of sufficiently large width product regions in $M-\bar{M}$ and let $T_{\alpha} \subset P_{\alpha}, \alpha \in A$, be the product regions from Step 2 that can be foliated by surfaces of bounded geometry. We want to show that there is a finite set $\left\{Q_{1}^{\prime}, \ldots Q_{s^{\prime}}^{\prime}\right\}$ such that when $M$ satisfies (*), $\widetilde{M}=\bar{M} \cup\left\{P_{\alpha}-T_{\alpha}\right\}_{\alpha \in A}$ satisfies the conditions of being the tiny manifold. First note that because $\# \pi_{0}(M-\bar{M}) \geqslant \# \pi_{0}(M-\widetilde{M})$, we have the same bound $r$ on the number of components in $\widetilde{M}$.

Note that there exists a uniform diameter bound $d$ on components of $\bar{M}$. Thus, any component of $\widetilde{M}$ has diameter bounded by $r(d+2 \delta)$, where $\delta$ is the largest diameter of a component of any $P_{\alpha}-T_{\alpha}$.

Suppose, for contradiction, that there exist manifolds $M_{i}$ satisfying $(*)$, each with some component $M_{i}^{0} \subset \overline{M_{i}} \cup\left\{\left(P_{i}\right)_{\alpha}-\left(T_{i}\right)_{\alpha}\right\}_{\alpha \in A}$, which together make up an infinite collection of distinct 2-bi-Lipschitz classes. Let $x_{i} \in M_{i}^{0}$. Since each $M_{i}$ is $\varepsilon$-thick, up to subsequence we have a geometric limit $\left(M_{i}, x_{i}\right) \rightarrow\left(M_{\infty}, x_{\infty}\right)$.

By taking the inverse of the maps, we get from the definition of geometric convergence, for large enough $i$ we have 2-bi-Lipschitz embeddings

$$
\varphi_{i}: M_{i}^{0} \longrightarrow M_{\infty}
$$

but they do not necessarily have the same image, so we have yet to arrive at a contradiction.

We need to change the image of the above maps in a controlled way. Start by fixing a particular boundary component in each $M_{i}^{0}$ and considering the sequence of maps:

$$
S_{g_{i}} \stackrel{\iota_{i}}{\longrightarrow} \partial M_{i}^{0} \stackrel{\varphi_{i}}{\longrightarrow} M_{\infty}
$$

where $S_{g_{i}} \in \mathcal{S}_{g \leqslant G}$. Up to subsequence, we can assume that the domains are the same: $S_{g}$ for some fixed $g$. Because all the maps are uniformly bi-Lipschitz with images in a compact region, they converge to some uniformly bi-Lipschitz map $\varphi_{\infty}: S_{g} \rightarrow M_{\infty}$. Note that because geometric convergence is actually smooth, we can assume that the maps $\iota_{i} \circ \varphi_{i}$ and their limit $\varphi_{\infty}$ are all smooth. As such, note that for large enough $i, \operatorname{im}\left(\iota_{i} \circ \varphi_{i}\right)$ lies in an arbitrarily small neighborhood of $\operatorname{im}\left(\varphi_{\infty}\right)$ and is transverse to its normal bundle.

Thus, for large enough $i$ we can find isotopies $\psi_{i}: M_{\infty} \rightarrow M_{\infty} \operatorname{taking} \operatorname{im}\left(\varphi_{i} \circ \iota_{i}\right)$ to $\operatorname{im}\left(\varphi_{\infty}\right)$ by locally stretching in the direction of the normal bundle of $\operatorname{im}\left(\varphi_{\infty}\right)$. By making sure such an isotopy is supported on a slightly larger neighborhood, we can choose the isotopies to be 2-biLipschitz. When we compose the $\varphi_{i}$ with associated 2-bi-Lipschitz isotopies described above, we arrive at a sequence of 4-bi-Lipschitz maps on the tail of the sequence $\varphi_{i} \circ \varphi_{i}: M_{i}^{0} \rightarrow M_{\infty}$, whose images agree on one boundary component.

We iterate this process by next fixing an unused boundary component in each $M_{i}^{0}$ and repeating the process. (We do not have to worry about distinct boundary components converging in the limit because the Hausdorff distance between any two boundary components of each $M_{i}^{0}$ is uniformly bounded from below.) We do this until we have, up to subsequence, bijections $M_{i}^{0} \rightarrow M_{\infty}^{0} \subset M_{\infty}$, where $M_{\infty}^{0}$ is compact. This will happen after a finite number of iterations because we have a uniform bound on the number of boundary components in each $M_{i}^{0}$. If the bound on the number of boundary components is $b$, the resulting maps will be $4^{b}$-bi-Lipschitz, meaning the $M_{i}^{0}$ fall into a finite number of $4^{b}$-bi-Lipschitz classes.

The next corollary follows almost immediately from Steps 1 and 2 in the proof of Theorem 10 . It is also the specific statement we will need to prove Theorem 1 in $\S 4$. 
Corollary 15. Given $\varepsilon, c>0$, there exist constants $p, G, V_{\min }, V_{\max }>0$ and $L>1$ such that for all but finitely many hyperbolic 3 -manifolds $M$ satisfying $(*)$ there exists a submanifold $T \subset M$ with the following properties:

(i) $\operatorname{vol}(T) \geqslant p \operatorname{vol}(M)$;

(ii) $T$ is homeomorphic to a product region $\Sigma_{g} \times I$ and $g \leqslant G$;

(iii) $T=T_{1} \cup \cdots \cup T_{n}$ with disjoint interiors, where each $T_{i}$ is $L$-bi-Lipschitz equivalent to $S_{g} \times[-1,1], S_{g} \in \mathcal{S}$

(iv) in particular, there exist $V_{\min }$ and $V_{\max }$ such that $V_{\min } \leqslant \operatorname{vol}\left(T_{i}\right) \leqslant V_{\max }$ for every $i$.

Proof. Because there are only finitely many components of the tiny manifold $\widetilde{M}$, and they come from a finite family of bi-Lipschitz classes, there is a bound on the number of components of $\partial \widetilde{M}$. In particular, there is an upper bound on the possible number of components of $M-\widetilde{M}$, call it $t$. Additionally, there is some upper bound on the volume of $\widetilde{M}$, call it $V$. Suppose $\operatorname{vol} M \geqslant$ $2 V$. Then there exists some component $T \subset M-\widetilde{M}$ such that $\operatorname{vol} T \geqslant \operatorname{vol} M / 2 t$. As we have noted before, there are only finitely many isometry classes of $\varepsilon$-thick hyperbolic 3 -manifolds of volume less than $2 \mathrm{~V}$.

The rest of the statements follow from the proof of Theorem 10.

\section{Proof of Theorem 1}

Recall our main result.

Theorem 1. For every $\varepsilon>0, c, k \in \mathbb{N}$, there exists $\Omega(\varepsilon, c, k)$ such that, if $M$ is a closed, $\varepsilon$-thick hyperbolic 3-manifold with $\operatorname{rank} \pi_{1}(M)<c$, then

$$
\frac{1}{\Omega \operatorname{vol}(M)^{2}} \leqslant \lambda_{k}(M) \leqslant \frac{\Omega}{\operatorname{vol}^{2}(M)},
$$

where $\lambda_{k}(M)$ is the $k$ th positive eigenvalue of the Laplace operator on $M$.

REMark. We use Corollary 15 for the following proof. Recall that Corollary 15 holds for all but finitely many $M$ satisfying $(*)$. It suffices to prove the upper bound in the theorem in this context because, for the finitely many $M$ not covered by Corollary 15, we can always find some $\Omega$ to satisfy the inequality by taking $\max \left\{\lambda_{k}\left(M_{\alpha}\right) \operatorname{vol}^{2}\left(M_{\alpha}\right)\right\}$ over a finite index set $\alpha \in A$.

Proof. As mentioned in $\S 1$, the lower bound is due to Schoen [15], because $\lambda_{k} \geqslant \lambda_{1}$.

To prove the upper bound, we use the 'max' half of the Minimax theorem. That is, for any set of functions $f_{0}, \ldots, f_{k}: M \rightarrow \mathbb{R}$ whose supports pairwise intersect with on zero-volume sets, we have $\lambda_{k} \leqslant \max \left\{R\left(f_{i}\right)\right\}_{i=0}^{k}$, where $R(f)=\int_{M}\|\nabla f\|^{2} / \int_{M} f^{2}$. We will define test functions piecewise on the decomposition $T=\bigcup_{i=1}^{n} T_{i}$ from Corollary 15.

Let $\varphi:[-1,1] \rightarrow[0,1]$ be a smooth function such that

(i) $\varphi(-1)=0$;

(ii) $\varphi(1)=1$; and

(iii) $\varphi$ is constant in some neighborhood of -1 and 1 .

Now define $\Phi: S_{g} \times[-1,1] \rightarrow[0,1]$ by $\Phi(x, t)=\varphi(t)$. From Corollary 15, we have $L$-biLipschitz embeddings $F_{i}: T_{i} \rightarrow S_{g} \times[-1,1]$. Thus, we have maps $\Phi \circ F_{i}: T_{i} \rightarrow[0,1]$. 


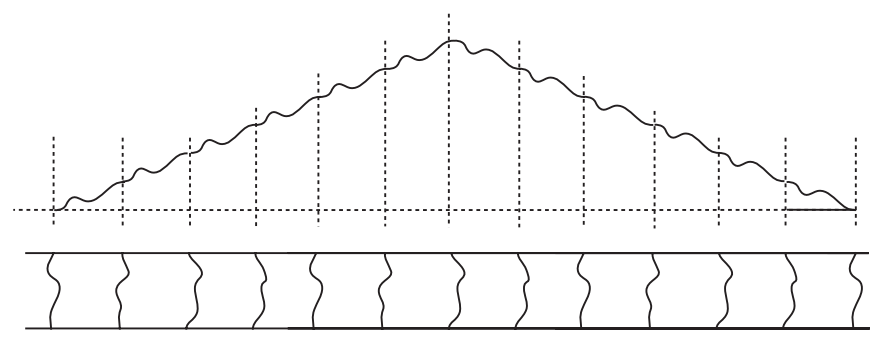

Figure 5. An example of $g_{\kappa}$ when $m=6$.

Recall that $\lfloor\star\rfloor$ denotes 'the integer part of $\star$ '. Let $m=\lfloor n / 2(k+1)\rfloor$, where $n$ is the number of pieces in $T$ from Corollary 15 . Note that $T$ can be decomposed coarsely into $k+1$ pieces (which will be the supports of our $k+1$ test functions), each consisting of $2 m$ consecutive $T_{i}$, that is,

$$
P_{0}=T_{1} \cup \cdots \cup T_{2 m}, \ldots, P_{k}=T_{2 k m+1} \cup \cdots \cup T_{2 m(k+1)},
$$

and a possibly empty piece $P_{\text {extra }}=T_{2 m(k+1)+1} \cup \cdots \cup T_{n}$. We will describe functions $g_{\kappa}$ supported on $P_{\kappa}, 0 \leqslant \kappa \leqslant k$, and then bound their Rayleigh quotients.

Roughly speaking, each $g_{\kappa}$ will grow to $m$ and decrease back down to 0 by increasing (or decreasing) by one on each $T_{i}$ using an appropriate translation of $\Phi \circ F_{i}$. More precisely, for $0 \leqslant \kappa \leqslant k$,

$$
g_{\kappa}(x)= \begin{cases}\Phi \circ F_{2 m \kappa+j}(x)+j, & x \in T_{2 m \kappa+j}, j \in[1, m], \\ -\Phi \circ F_{2 m \kappa+j}(x)+2 m-j+1, & x \in T_{2 m \kappa+j}, j \in[m+1,2 m] .\end{cases}
$$

To see an example of $g_{\kappa}$ when $m=6$, refer to Figure 5. Also realize that all the $g_{\kappa}$ are 'copies' of $g_{0}$ defined on the appropriate $P_{\kappa}$.

Recall that our goal is to bound $R\left(g_{\kappa}\right)=\int_{M}\left\|\nabla g_{\kappa}\right\|^{2} d M \int_{M} g_{\kappa}^{2} d M$ for each $0 \leqslant \kappa \leqslant k$. On the $T_{i}$ where $g_{\kappa}$ is non-zero, the following holds by the chain rule:

$$
\begin{aligned}
\int_{T_{i}}\left\|\nabla g_{\kappa}\right\|^{2} d M & =\int_{F_{i}\left(T_{i}\right)}\left\|\nabla\left(g_{\kappa} \circ F_{i}^{-1}\right)\right\|^{2}\left(F_{i_{*}} d T_{i}\right) \\
& =\int_{S_{g} \times[-1,1]}\|\nabla \Phi\|^{2}\left(F_{i_{*}} d T_{i}\right) \\
& \leqslant L^{3} \int_{S_{g} \times[-1,1]}\|\nabla \Phi\|^{2} d\left(S_{g} \times[-1,1]\right),
\end{aligned}
$$

where $L^{3}$ factor comes from the fact that each $F_{i}$ is an $L$-bi-Lipschitz embedding and our volume forms are 3 -forms. Thus,

$$
\int_{M}\left\|\nabla g_{\kappa}\right\|^{2} d M=\int_{P_{\kappa}}\left\|\nabla g_{\kappa}\right\|^{2} d M \leqslant 2 m L^{3} \int_{S_{g} \times[-1,1]}\|\nabla \Phi\|^{2} d\left(S_{g} \times[-1,1]\right) .
$$

Also note that

$$
\int_{M} g_{\kappa}^{2} d M=\int_{T} g_{\kappa}^{2} d M \geqslant 2 V_{\min } \sum_{i=1}^{m}(m-1)^{2} \geqslant V_{\min } m^{3} / 6,
$$

by definition of $g_{\kappa}$. 
Now we can calculate:

$$
\begin{aligned}
R\left(g_{\kappa}\right) & =\frac{\int_{M}\left\|\nabla g_{\kappa}\right\|^{2} d M}{\int_{M} g_{\kappa}^{2} d M} \\
& \leqslant \frac{2 m L^{3} \int_{S_{g} \times[-1,1]}\|\nabla \Phi\|^{2} d\left(S_{g} \times[-1,1]\right)}{V_{\min } m^{3} / 6} \\
& \leqslant \frac{K^{\prime}}{m^{2}},
\end{aligned}
$$

where $K^{\prime}$ depends only on $\varepsilon, k$, and $c$. Lastly, note that $m \geqslant n / 2 k, n \geqslant \operatorname{vol}(T) / V_{\max }$, and by Corollary 15 , there exists $p$ such that $\operatorname{vol}(T) \geqslant p \operatorname{vol}(M)$. Thus,

$$
m \geqslant \frac{p \operatorname{vol} M}{2 k V_{\max }} \text {. }
$$

With that, Theorem 1 is proved.

\title{
References
}

1. R. Benedetti and C. Petronio, Lectures on hyperbolic geometry, Universitext (Springer, Berlin, 1992).

2. I. Biringer, 'Geometry and rank of fibered hyperbolic 3-manifolds', Algebr. Geom. Topol. 9 (2009) 277-292.

3. I. Biringer and J. Souto, 'A finiteness theorem for hyperbolic 3-manifolds', J. London Math. Soc. (2) 84 (2011) 227-242.

4. F. Bonahon, 'Bouts des variétés hyperboliques de dimension 3', Ann. of Math. (2) 124 (1986) 71-158.

5. M. Burger and R. D. CANARY, 'A lower bound on $\lambda_{0}$ for geometrically finite hyperbolic $n$-manifolds', J. reine angew. Math. 454 (1994) 37-57.

6. P. Buser, Geometry and spectra of compact Riemann surfaces, Modern Birkhäuser Classics (Birkhäuser Boston, Boston, MA, 2010). Reprint of the 1992 edition.

7. R. D. Canary, 'On the Laplacian and the geometry of hyperbolic 3-manifolds', J. Differential Geom. 36 (1992) 349-367.

8. J. Cheeger, 'A lower bound for the smallest eigenvalue of the Laplacian', Problems in analysis (Papers dedicated to Salomon Bochner, 1969) (Princeton University Press, Princeton, NJ, 1970) 195-199.

9. B. Colbois and J. Dodziuk, 'Riemannian metrics with large $\lambda_{1}$,' Proc. Amer. Math. Soc. 122 (1994) 905-906.

10. J. Dodziuk and B. RAndol, 'Lower bounds for $\lambda_{1}$ on a finite-volume hyperbolic manifold', J. Differential Geom. 24 (1986) 133-139.

11. J. Hempel, '3-Manifolds', Annals of Mathematics Studies 86 (Princeton University Press, Princeton, NJ, 1976).

12. H. Huber, 'Über den ersten Eigenwert des Laplace-Operators auf kompakten Riemannschen Flächen', Comment. Math. Helv. 49 (1974) 251-259.

13. D. D. Long, A. Lubotzky and A. W. Reid, 'Heegaard genus and property $\tau$ for hyperbolic 3-manifolds', J. Topol. 1 (2008) 152-158.

14. K. Matsuzaki and M. Taniguchi, Hyperbolic manifolds and Kleinian groups, Oxford Mathematical Monographs, Oxford Science Publications (The Clarendon Press, Oxford University Press, New York, 1998).

15. R. Schoen, 'A lower bound for the first eigenvalue of a negatively curved manifold', J. Differential Geom. 17 (1982) 233-238.

16. W. P. Thurston, Three-dimensional geometry and topology, vol. 1, Princeton Mathematical Series 35 (ed. S. Levy; Princeton University Press, Princeton, NJ, 1997).

17. H. C. WANG, 'Topics on totally discontinuous groups', Symmetric spaces (Short Courses, Washington Univ., St. Louis, Mo., 1969-1970), Pure and Applied Mathematics 8 (Dekker, New York, 1972) 459-487.

\author{
Nina White \\ Department of Mathematics \\ University of Michigan \\ 530 Church St. \\ Ann Arbor, MI 48109 \\ USA
}

whitenj@umich.edu 